\title{
"Endometriosis-Like Syndrome": from the concept of Painful Uterine Syndrome to the use of Myometrial Botulinum Toxin injections under hysteroscopy.
}

\author{
ERIC BAUTRANT ${ }^{1}$, OONA FRANKE ${ }^{1}$, Christophe AMIEL ${ }^{1}$, Thierry BENSOUSAN ${ }^{1}$, \\ Dominique THIERS-BAUTRANT ${ }^{1}$, and Christine LEVÊQEE ${ }^{2}$ \\ ${ }^{1}$ Clinique Axium-Centre L'Avancée \\ ${ }^{2}$ Clinique Axium-Centre L'Avancée
}

May 27, 2020

\begin{abstract}
Background: A new concept is being advanced to explain undiagnosed chronic pelvic pain, severe dysmenorrhoea, painful uterine contractions, and deep dyspareunia in women, which has been shown to be anatomically negative. Search strategy: In these "endometriosis-like" syndrome situations, there are many pathophysiological arguments supporting the existence of a visceral uterine sensitization syndrome. Data collection and analysis: These arguments are further developed and use central sensitization mechanisms, just like those for irritable bowel syndrome (IBS) or painful bladder syndrome (PBS). The name "painful uterine syndrome" (PUS) has been suggested, and a basis for a PUS diagnostic score has been established. Central sensitization and visceral sensitization mechanisms have been developed. Muscular hypertonia is common in condition of central sensitization and increased uterine contractility has been confirmed using cine magnetic resonance imaging in PUS situations. These findings led to the application of botulinum toxin (BTX) injections under hysteroscopy of the uterine myometrium in PUS. Main results: A pilot study reported the first use of BTX uterine injections under hysteroscopy in PUS and this pilot study has been analysed. It focuses on a simple and reproducible treatment procedure which shows improvement in dysmenorrhoea and dyspareunia as well as improved quality of life scores. Conclusions: Only long-term randomised studies will be able to confirm that BTX injections are useful as a treatment for this condition. The randomised long-term study, Uteroxine, started in 2020 and will shortly release its results.
\end{abstract}

\section{Introduction:}

For the past century, countless attempts have been made to find physio-pathological explanations for chronic pelvic pain in women as well as for the severe and disabling forms of dysmenorrhoea that can lead to absence from school or even work, often with serious socio-economic consequences (1).

Research on this subject began in the 1950s with Allen and Masters' theory on tears in the large ligament (2). Then came the role of uterine retroversion in the 1960s (3), the effect of intra-peritoneal adhesions from the 1970s (4), the effect of prostaglandins in the 1980s (5), the possible role of pelvic varicose veins in the 1990s (6), Petros' theory of ligament laxity (7) from the 2000s, and finally, more recently, endometriosis (8) which is now widely mentioned, despite a long period of disinterest.

However, although endometriosis is one of the major underlying causes of chronic pelvic pain and severe dysmenorrhoea, it does not explain all of the symptoms.

\section{Methods :}


Observational studies (9) have highlighted many situations in which endometriosis fails to explain certain symptoms:

- In most cases, all images and laparoscopies provide normal results in cases of very severe primary dysmenorrhoea in young girls.

- Many patients are seen for endometriosis-like symptoms, but all examinations, images, and laparoscopies appear normal.

- Other patients who have undergone complete surgical treatment for their endometriosis continue to have the same "endometriosis-like" symptoms.

- Some patients who have recovered from their painful symptoms following treatment for endometriosis, return for a suspected endometriosis recession when pelvic pain and dysmenorrhoea recur, but no endometriosis is found.

- One report concerns patients complaining of unexplained deep dyspareunia with a painful uterus, even though the examinations, images, and laparoscopies provided normal results.

These different findings suggest that we need to find an alternative explanation, consider a paradigm shift, and may even need to rethink the entire issue.

\section{The concept of Painful Uterine Syndrome (PUS):}

It is based on the principle of central sensitization, described by Woolf in 1983 (10).

Central sensitization is defined as a decrease in cortical nociceptive thresholds (10-13). This is responsible for a variety of problems such as neuropathic pain, muscle spasms, autonomic nervous system disorders, and visceral sensitization.

Abdominal visceral sensitization is known to occur in irritable bowel syndrome (14-17). The same applies at the pelvic level, where painful bladder syndrome (PBS) also appears to be related to the central sensitization mechanisms (18-21).

The existence of uterine sensitization is, therefore, very likely. This hypothesis has become increasingly evident in recent years, with publications by Iacovides in 2013 (22) and 2015 (23), Giamberardino in 2016 (24), and finally, Jarell in 2016 (25 and 26), which confirm a significant reduction in central pain thresholds in dysmenorrheic patients.

The concept of PUS allows us to better understand pelvic pain, whose symptomatic pattern appears to be uterine, with a negative anatomical evaluation, associating primary dysmenorrhoea and painful symptoms related to episodes of violent and inappropriate uterine myometrial contractions. Muscle hypercontractility is a common occurrence in sensitization. Several cine magnetic resonance (cine MRI) studies confirm the significant increase in uterine myometrial peristalsis in dysmenorrheic patients (27-29).

In 2011, we published a diagnostic score for uterine adenomyosis (30), which allowed for a positive diagnosis with a sensitivity of $98 \%$ and specificity of $90 \%$. This diagnostic score is usefully applied in PUS cases (Table 1) and is defined by a particular type of complaint where the pain, located in the pelvis, is typical for uterine pain, including cramping and contractions, dysmenorrhoea, and deep dyspareunia. A physical examination reveals a painful uterine pain trigger that reproduces the pain.

The concept of PUS not only provides us with a better understanding of the painful symptoms, but it also allows us to better understand how to treat these symptoms and the treatment options available. This validates the suppression of menstrual function as one of the important, primary focus areas in the treatment of dysmenorrhoea, whether or not it is caused by endometriosis (24 and 25).

If this fails, there are few conservative treatment options available.

The logical connection with PBS and overactive bladder (OAB), as well as the importance of myometrial hypercontractility mechanisms led us to consider the use of Botulinum Toxin (BTX) injections for treating PUS. BTX injections have been shown to be effective in patients with an overactive bladder, where detrusor 
hypercontractility is the main symptom (31). While BTX's effectiveness is somewhat less evident in PBS, it is an attractive treatment option that has been confirmed by recent randomised controlled studies or meta-analysis (32 and 33).

\section{Uterine Myometrium Botulinum Toxin A (BTX) Injections.}

The principle is similar to that proposed for BTX injections in the bladder detrusor under cystoscopy for OAB.

The BTX units are evenly distributed in the anterior and posterior myometrial wall under hysteroscopic control.

In 2018, we conducted a prospective feasibility study on 30 patients with severe dysmenorrhoea. The technique and results were presented at the International Neurotoxin Association - INA's 2019 International Toxins Conference (34) and at the "Convergences PP" annual congress in Madrid in November 2019 (35).

Uterine myometrial BTX injections are performed under hysteroscopy as an outpatient procedure using a short-term general anaesthetic. The $5 \mathrm{~mm}$ Bettocchi type hysteroscope has an operating channel for the needle to pass through.

The effective dose used in this study was 200 IU of BTX, Incobotulinum-toxin A.

The $200 \mathrm{IU}$ of BTX is diluted in saline and evenly distributed in the uterine myometrium with 10 injections in each anterior and posterior muscle wall under hysteroscopic control (figure 1).

All patients fulfilled the PUS criteria and ranged from 17 to 44 years old.

Severe dysmenorrhoea was found in 24 of the patients (89\%), all patients had a painful uterine trigger at vaginal examination and 2 patients had deep dyspareunia as the main symptom.

A pelvic ultrasound and MRI ruled out pelvic pathology and endometriosis.

All patients had already received initial treatment such as painkillers, anti-inflammatory drugs, the contraceptive pill, and menstrual suppressant therapy.

Pregnancy was contraindicated during the 4 months following the injection.

Patients were reviewed at 8 weeks, 12 weeks, and 6 months.

They were then assessed on the level of pain using the Visual Analogue Scale (VAS) and the Quality of Life Questionnaire, using the Medical Outcomes Study SF-36 (MOS SF-36), validated in French. All patients were screened for co-morbidities and central pelvic hypersensitivity criteria using the Convergences PP criteria (36).

In terms of statistical analyses, the pre and post-treatment groups were compared using the Freidman test for multiple, dependant non-parametric data. P-values were two tailed and significance was set at the $5 \%$ level.

\section{Results :}

No patients were lost during follow-up.

Very few side effects were reported: 2 patients $(6.7 \%)$ experienced a severe worsening of their pain in the immediate post-operative period. Both patients were then significantly improved by their first follow-up visit at 8 weeks.

VAS scores were significantly improved (Table 2 ) for dysmenorrhoea ( 80 vs $25: \mathrm{p}=0.001$ ), dyspareunia (71 vs 28: $\mathrm{p}=0.02$ ), and pelvic pain outside of menstruation ( $67 \mathrm{vs} 28: \mathrm{p}=0.03$ ). Quality of life scores all improved dramatically. The sexual activity questionnaire scores also improved, with a significantly reduced level of discomfort (4.8 vs. 2.2: $\mathrm{p}=0.02)$ and improvement in habit (0,2 vs 1,9: $\mathrm{p}=0,03)$. 9 patients (30\%) had positive criteria for pelvic hypersensitivity. In this group, the improvement was not significant for all scores. 
In this study's follow-up, $39 \%$ of patients were given new injections before the reappearance of PUS.

\section{Discussion:}

The principle of central sensitization, as described by Woolf in 1983, is a manifestation of the somatosensory plasticity of the central nervous system $(10,37)$.

Central nociceptive sensitization develops as a result of sustained nociceptor activity in physiological conditions but also in pathological situations following inflammatory attacks, nerve damage, or trauma (38).

The mechanisms involved in the development of central nociceptive sensitization are quite complicated. They involve disrupting inter and intracellular neuronal signalling using numerous membrane receptors and various intracellular transduction pathways, resulting in changes in genetic expressions.

Changing cell phenotypes is one aspect of maintaining central nociceptive sensitization (38,39 and 40). A growing number of studies indicate that epigenetic changes may be involved in pathophysiology (41).

Woolf summarises central sensitization as the lowering of cortical nociceptive thresholds (10-13). These lower pain thresholds will have multiple clinical consequences and lead to so-called co-morbidities $(11,24)$. Therefore, myofascial pain, autonomic disorders with hyperalgesia, urinary, rectal and sexual dysfunction, neuropathic pain, and visceral pain are more or less associated (42).

\section{Main Findings :}

The existence of PUS by central sensitization, as defined above, which in particular causes severe dysmenorrhoea, appears increasingly plausible, regardless of whether endometriosis is present or not. Recent studies by Evans (43), Stratton (44), and Grundström (45) all point in this direction.

PUS or "endometriosis-like" syndrome is typically identified by negative imaging and anatomical findings. The main symptom is severe dysmenorrhoea; however, pelvic pain from uterine contractions, deep dyspareunia, and uterus trigger pain at vaginal examination, are among the diagnostic criteria as defined in the PUS diagnostic score (Table 1).

The Viscero-Visceral sensitization mechanisms, as described by Giamberardino (46), account for a common association with painful bladder syndrome through peripheral sensitization phenomena. Similarly, comorbidities such as hyperesthesia of the vulvar vestibule, are connected with the occurrence of the frequent Provoked Vulvar Vestibulodynia (PVD) (47), which is responsible for introductory dyspareunia.

But the most frequently associated disorders are pelvic floor muscle hypertonia and myofascial syndromes $(42,44)$. This muscle hypertonia is consistent with pelvic sensitization and, therefore, applies to PUS, which has been well documented in the various cine MRI studies, as described above.

In terms of therapeutic consequences, BTX injections have a logical application here. Besides its widely demonstrated effectiveness in treating muscle spasms, hypertonia, or hyperactivity by inhibiting the release of acetylcholine at the neuromuscular junction by competitive neurotoxin inhibition (48 and 49), BTX may also have direct peripheral and central analgesic effects. It may also have a direct effect on inflammation by reducing the release of pro-inflammatory neuropeptides (50 and 51)

The use of BTX in pelvic floor muscle hypertonia, spasms, and myofascial pain was first documented by Abbott in 2006 (52). Several publications have confirmed that botulinum toxin injections are useful for treating this condition $(53,54,55,57$, and 58). The effectiveness of BTX injections on pelvic floor muscle hypertonia and spasms has been proven in a number of prospective and randomised studies (54 \& 57 ). On the other hand, for myofascial pain, the randomised studies conducted by Dessie (59) and Levesque (60) found no significant difference with the control group for saline and local anaesthetics, respectively.

As far as we know, uterine myometrial BTX injections used in cases of PUS and/or severe dysmenorrhoea has never been described before. This indication follows the same pathophysiological approach as that proposed for $\mathrm{OAB}$ or PBS $(31,32 \& 33)$. 
The procedure under hysteroscopy is simple and can be easily reproduced. Our pilot study (34), mentioned above, reported no immediate or remote post-operative complications.

Although pregnancy was not recommended within 4 months of the myometrial injection in this study, no maternal or fetal complications were reported in the literature when a botulinum toxin injection was administered during pregnancy ( $61 \& 62)$. One patient in our study became pregnant, despite being advised against it. This was an unintended pregnancy and a voluntary termination was performed in the first trimester. This procedure was carried out without any complications.

\section{Strengths and Limitation :}

Our study demonstrated significant improvements in PUS, dysmenorrhoea, and deep dyspareunia in patients without co-morbidities or Convergences PP pelvic sensitization criteria (36). The best results were obtained when the uterine pain symptoms were isolated, with no associated pelvic floor myofascial syndrome or co-morbidities (PBS or PVD).

In our group of patients with pelvic sensitization criteria, there was no significant improvement in the quality of life scores, but the Central Sensitization Inventory (CSI) (63) improved for more than half of the patients in this group.

The results of this pilot feasibility study suggest an interesting perspective for the treatment of severe dysmenorrhoea and PUS, in the absence of alternative treatment, and in cases where well-conducted medical treatments have failed.

A prospective, randomised, double-blind, multicentric study (Uteroxine) is currently underway which compares the hysteroscopic myometrial injection (200 IU of botulinum toxin A) to the $2 \mathrm{mg} / \mathrm{ml}$ Ropivacaine injection.

The results of the Uteroxine randomised comparative study should allow us to confirm the benefits of botulinum toxin injections as a treatment for this condition.

\section{Conclusion:}

A significant amount of physio-pathological evidence supports the existence of visceral uterine sensitization syndrome.

We call it Painful Uterine Syndrome (PUS).

It can be used to explain a series of pelvic pain symptoms that were previously difficult to explain in women, including severe dysmenorrhoea with a negative anatomical assessment.

Diagnostic criteria specific to PUS, which are proposed here with satisfactory sensitivity, could be made available in order to facilitate diagnosis.

PUS may be associated with PBS and irritable bowel syndrome through peripheral viscero-visceral sensitivity mechanisms.

However, the phenomenon most frequently associated with visceral sensitization is muscular hypercontractility. This is demonstrated in the uterine myometrium, opening the way for treatment with BTX injections.

A pilot study reporting the first use of BTX injections under hysteroscopy in PUS indicated an improvement in dysmenorrhoea and dyspareunia as well as improved quality of life scores. The prospective and randomised Uteroxine study is currently underway to confirm the necessary initial results.

DISCLOSURE OF INTERESTS : the authors do not declare any conflict of interest.

ETHICS STATEMENT: No ethics approval was necessary for this work.

FUNDING: No funding was necessary for this work. 
CONTRIBUTION TO AUTHORSHIP: EB and CL were at the beginning of the concept of the Painful Uterine Syndrome and the application to the botulinum toxin injection into the uterine myometrium. EB was the main writer with the close help of OF, who made the corrections, the tables and the figure. CA, CL and DTB were in charge to review the research on central sensitization and of the references. TB is the anesthesiologist and pain specialist of the team, in charge of the pain evaluation, questionnaire and pain medications.

\section{REFERENCES}

1. Ju H, Jones M, Mishra G. The prevalence and Risk Factors of Dysmenorrhea. Epidemiol Rev 2014;36 (1):104-113.

2. Allen WM, Masters WH. Traumatic laceration of uterine support. Am J Obstet Gynecol. 1955;70:50013.

3. Cagnacci A, Grandi G, Cannoletta, Xholli A, Piacenti I, Volpe A. Intensity of menstrual pain and estimated angle of uterine flexion. Acta Obstet Gynecol Scand 2014 Jan;93(1):58-63.

1. Hammoud A, Gago LA, Diamond MP. Adhesions in patients with Chronic Pelvic pain : a role for adhesiolysis? Fertil Steril 2004 Dec;82(6):1483-91.

2. Chan WY. Prostaglandins and nonsteroidals antiinflammatory drugs in dysmenorrhera. Annu Rev Pharmacol Toxicol 1983;23:131-149.

3. Borghi C, Dell'Atti L. Pelvic congestion syndrome : the current state of the literature. Arch Gynecol Obstet 2016 Feb;293(2):291-301.

4. Petros PE, Richardson PA. TFS posterior sling improves overactive bladder, pelvic pain and abnormal emptying, even with minor prolapse-a prospective urodynamic study. Pelviperineology 2010;29:52-5.

5. Stratton P, Berkley KJ. Chronic Pelvic Pain and endometriosis: translational evidence of the relationship and implications. Human Reprod Update 2011 May-Jun;17(3):327-46.

6. López A. Pain and Endometriosis. Convergences PP annual congress; Madrid 2019 November 14-16th.

7. Woolf CJ. Evidence for a central component of post-injury pain hypersensitivity. Nature 1983;3065944:686-8.

8. Woolf CJ. Central Sensitization : uncovering the relation between pain and plasticity. Anesthesiology 2007;106(4):864-7.

9. Woolf CJ. Central Sensitization : Implications for the diagnosis and treatment of pain. Pain 2011 ; 152(suppl 3):S2-15.

10. Woolf CJ. What to call amplification of nociceptive signals in the central nervous system that contribute to wispread pain. Pain 2014;155(10):1911-2.

11. Bonaz B. Visceral sensitivity perturbation integration in the brain-gut axis in functional digestive disorders. J Physiol Pharmacol 2003 Dec ;54 Suppl 4:27-42.

12. Kanazawa M, Hongo M, Fukudo S. Visceral hypersensitivity in irritable bowel syndrome. J Gastroenterol Hepatol 2011 Apr;26 Suppl 3:119-21.

13. Eisenbruch S. Abdominal pain in irritable bowel syndrome : a review of putative psychological, neural and neuro-immune mechanisms. Brain Behav Immun 2011 Mar;25(3):386-94.

14. Keszthelyi D, Troost FJ, Simrén M, Ludidi S, Kruimel JW, Conchillo Jm et al. Revisiting concepts of visceral nociception in irritable bowel syndrome. Eur J Pain 2012 Nov;16(10):1444-54.

15. Fitzgerald MP, Koch D, Senka J. Visceral and cutaneous sensory testing in patients with painful bladder syndrome. Neurourol Urodyn 2005;24(7):627-32.

16. Lai HH, Gardner V, Ness TJ, Gereau RW. Segmental hyperalgesia to mechanical stimulus in interstitial cystitis/bladder pain syndrome : evidence of central sensitization. J Urol 2014 May;191(5):1294-9.

17. Grundy L, Caldwell A, Brierley SM. Mechanisms underlying overactive bladder and interstitial Cystitis/painful bladder syndrome. Front Neurosci 2018 Dec 12;12:931.

18. Akiyama Y, Luo Y, Hanno PM, Maeda D, Homma Y. Interstitial cystitis/bladder pain syndrome : The evolving landscape, animal models and future perspectives. Int J Urol 2020 Apr 4. Doi :10.

19. Iacovides S, Baker FC, Bentley A. Women with dysmenorrhea are hypersensitive to experimental deep muscle pain across the menstrual cycle. J Pain 2013 Oct;14(10):1066-76. 
20. Iacovides S, Avidon I, Baker FC. What we know about primary dysmenorrhea today : a critical review. Hum Reprod Update 2015 Nov-Dec;21(6):762-78.

21. Giamberardino MA, Tana C, Costantini R. Pain theresholds in women with chronic pelvic pain. Curr Opin Obstet Gynecol 2014 Aug;26(4):253-9.

22. Jarrell J, Arendt-Nielsen J. Allodynia and dysmenorrhea. J Obstet Gynecol Can 2016 Mar;38(3):270-4.

23. Jarrell J, Arendt-Nielsen J. Evolutionary considerations in the development of chronic pelvic pain. Am J Obstet Gynecol. 2016 Aug;215(2):201.e1-4.

24. Katoaka M, Togashi K, Kido A, Fujiwara T, Koyama T, Fujii S. Dysmenorrhea : evaluation with cine-mode-display MR imaging initial experience. Radiology 2005 Apr;235(1):124-31.

25. Guo SW, Mao X, Ma Q, Liu X. Dysmenorrhea and its severity are associated with increased uterine contractility and overexpression of oxytocin receptor (OTR) in women symptomatic adenomyosis. Fertil Steril 2013 Jan;99(1):231-40.

26. Liu S, Zhang Q, Yin C, Chan Q, Chen W, He J, Zhu B. Optimized approach to cine MRI of uterine peristalsis. J Magn Reson Imaging 2016 Dec ;44(6) :1397-1404.

27. Bautrant E, Bryselbout MA, Thiers-Bautrant D, Eggermont E, Boyer R, Cassin H et al. Chronic pelvic pain syndrome due to uterine adenomyosis : diagnostic criteria and conservative treatment. Pelvi Perineol 2011;6:42-45.

28. Reynolds WS, Dmochowski R, Wein A, Bruehl S. Does central sensitization help explain idiopathic overactive bladder ? Nat Rev Urol 2016 Aug;13(8) :481-91.

29. Kuo HC, Jiang YH, Tsai YC, Kuo YC. Intravesical botulinum toxin-A injections reduce bladder painof interstitial cystitis/bladder pain syndrome refractory to conventional treatment - A prospective, multicenter, randomized, double-blind, placebo-controlled clinical trial. Neurourol Urodyn 2016 Jun;35(5):609-14.

30. Giannantoni A, Gubbiotti M, Bini V. Botulinum Neurotoxin A intravesical injections in interstitial cystitis/bladder painful syndrome : A systematic review with meta-analysis. Toxins (Basel) 2019 Sep $30 ; 11(9): 510$.

31. Bautrant E, Levêque C, Boyer R, Collet S, Bartoli D, Carbone P. Pilot study of Botulinum Toxin A uterine muscle injection in the the treatment of acute dysmenorrhea, uterine pain and deep dyspareunia. Toxicon 2018;S2-S120:S7-8.

32. Bautrant E. « Endometriosis like $\gg$ syndrome : from the concept of uterine pain syndrome to the use of Botulinic Toxin injections. Convergences PP annual congress ; Madrid 2019 November 14-16th.

33. Levesque A et al, Convergences PP Network. Cinical criteria of central sensitization in chronic pelvic and perineal pain (Convergences PP criteria): elaboration of a clinical evaluation tool based on formal expert consensus. Pain Med 2018;19(10):2009-2015.

34. Latremoliere A, Woolf CJ. Central sensitization : a generator of pain hypersensitivity by central neural plasticity. J Pain 2009 sep10;(9):895-926.

35. Pohl M Central nociceptive sensitization : cellular and molecular signalization systems. Douleurs Evaluation-Diagnostic-Traitement 2014;15:44-51.

36. Sengupta JN. Visceral pain : the neurophysiological mechanism.Handb Exp Pharmacol 2009;194:32-74.

37. Janig W. Neurobiology of visceral pain. Schmerz 2014 Jun;28(3):233-51.

38. Zang Z, Cai YQ, Zou F, Bie B, Pan ZZ. Epigenetic suppression of GAD65 expression mediates persistant pain. Nat Med 2011;17:1448-55.

39. Affaitati G, Costantini R, Tana C, Cipollone F, Giamberardino MA. Co-occurrence of pain syndromes. J Neural Transm (Vienna) 2020 Apr;127(4):625-646

40. Evans SF, Brooks TA, Esterman AJ, Hull ML, Rolan PE. The comorbidities of dysmenorrhea : a clinical survey comparing symptom profile in woman with and without endometriosis. J Pain Res 2018 Dec 13;11:3181-3194.

41. Stratton P, Khachikyan I, Sinaii N, Ortiz R, Shah j. Association of chronic pelvic pain and endometriosis with signs of sensitization and myofascial pain. Obstet Gynecol 2015 Mar;125(3):719-28.

42. Grundström H, Gerdle B, Alehagen S, Berterö C, Arendt-Nielsen L et al. Reduced pain thersholds and signs of sensitization in women with persistent pelvic pain and suspected endometriosis. Acta Obstet 
Gynecol Scand 2019 Mar;98(3):327-336.

43. Giamberardino MA, Costantini R, Affaitati G, Fabrizio A, Tafuri E, Mezzetti A. Viscero-visceral hyperalgesia: characterization in different clinical models. Pain 2010 Nov;151(2):307-22.

44. Bautrant E, O Porta, F Murina, H Mühlrad, C Levêque, T Riant, S Ploteau, G Valancogne, A Levesque. Provoked vulvar vestibulodynia : Epidemiology in Europe, physio-pathology, consensus for first line treatment and evaluation of second-line treatments. J Gynecol Obstet Hum Reprod 2019;48:685-88.

45. Poulain B. Botulinum neurotoxin. Rev Neurol(Paris). 2010 Jan;166(1):7-20.

46. Montal M. Botulinum neurotoxin : a marvel of protein design. Annu Rev Biochem 2010;79:591-617.

47. Pellett S, Yaksh TL, Ramachandran R. Current status and future directions of botulinum neurotoxins for targeting pain processing. Toxins (Basel) 2015;7(11):4519-4563.

48. Aredo JV, Heyrana KJ, Karp BI, Shah JP, Stratton P. Relating chronic pelvic pain and endometriosis to signs of sensitization and myofascial pain and dysfunction. Semin Reprod Med 2017 Jan;35(1):88-97.

49. Abbott JA, Jarvis SK, Lyons SD, Thomson A, Vancaille TH. Botulinum toxin type A for chronic pain and pelvic floor spasm in women : a andomized controlled trial. Obstet Gynecol 2006 Oct;108(4):915-23.

50. Bautrant E, Quintas-Marquès L, Eggermont J, Faller E, Weber P, Poirson E, Boyer R, Carbone P. Use of botulinum toxin neurotoxin type A injections to the perineal muscles in cases of perineal pain and dysfunction associated with pelvic floor muscle overcontraction and myalgia. Toxicon 2014;11:021.

51. Morrissey D, El-Khawand D, Ginzburg N, Wehbe S, O'Hare P3rd, Whitmore K. Botulinum toxin A injections into pelvic floor muscles under electromyographic guidance for women with refractory hightone pelvic floor dysfunction : A 6-month prospective pilot study. Female Pelvic Med Reconstr Surg 2015 Sep-Oct;21(5):277-82.

52. Karp BI, Tandon H, Vigil D, Stratton P. Methodological approaches to botulinum toxin for the treatment of chronic pelvic pain, vaginismus, and vulvar pain disorders. Int Urogynecol J 2019 Jul;30(7):1071-1081.

53. Tandon HK, Stratton P, Sinaii N, Shah J, Karp BI. Botulinum toxin for chronic pelvic pain in women with endometriosis : a cohort study of a pain-focused treatment. Reg Anesth Pain Med 2019 Jul 8 (ahead of print).

54. Abbott J. Gynecological indications for the use of botulinum toxin in women with chronic pelvic pain. Toxicon 2009 Oct:54(5):647-53.

55. Bhide AA, Puccini F, Khullar V, Elneil S, Digesu GA. Botulinum neurotoxin type A injection of the pelvic floor muscle in pain due to spasticity : a review of the current literature. Int Urogynecol J. 2013 Sep;24(9):1429-34.

56. Dessie SG, Von Bargen E, Hacker MR, Haviland MJ, Elkadry E. A randomized, double blind, placebocontrolled trial of onabotulinumtoxin A trigger point injections for myofascial pelvic pain. Am J Obstet Gynecol 2019 Nov;221(5):517.

57. Levesque A, Ploteau S, Michel F, Siproudhis L, Bautrant E, Eggermont J, Rabischong B, Volteau C, Perrouin-Verbe MA, Labat JJ. Botulinum toxin infiltrations versus local anaesthetic infiltrations in pelvic floor myofascial pain : Multicentre, randomized, double blind study. Ann Phys Rehabil Med 2020 Jan 22 (ahead of print).

58. Brin MF, Kirby RS, Slavotinek A, Miller-Messana MA, Parker L et al. Pregnancy outcomes following exposure to onabotulinumtoxinA. Pharmacoepidemiol Drug Saf 2016 Feb;25(2):179-87.

59. Li W, Tang M. Application of botulinum toxin in pregnancy and its impact in female reproductive health. Expert Opin Drug Saf 2020 Jan;19(1):83-91.

60. Neblett R, Hartzell MM, Cohen H, Mayer TG, Williams M, Choi Y et al. Ability of the Central Sensitization Inventory to Identify Central Sensitivity Syndromes in an Outpatient Chronic Pain Sample. Clin J Pain 2014;31(4) :323-32.

\section{TABLE 1 : CRITERIA FOR PAINFUL UTERINE SYNDROME}

Diagnostic score: positive when the score is [?] $5+$ imaging and/or laparoscopy eliminating uterine pathology or endometriosis. $98 \%$ sensitivity and $90 \%$ specificity. 


\section{CRITERIA}

Location : Chronic pelvic pain in the lower abdomen (hypogastric)

Type : cramps, uterine contraction sensations, heaviness. Experiencing menstrual pain outside of the menstrual period

Dysmenorrhoea I or II with or without associated

hypermenorrhoea

Deep Dyspareunia

Vaginal examination: painful uterine trigger

Associated co-morbidities: PVD, Pelvic floor muscle myofascial syndrome, PBS, IBS, Pelvic Sensitization criteria

TOTAL

\section{SCORE}

1

2

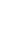

2

2

1

$/ 10$

TABLE 2 : Changes in pain symptoms and quality of quality of life 8 to 12 weeks after uterine myometrial botulinum toxin injections (34)

\section{Hosted file}

image1.emf available at https://authorea.com/users/326707/articles/454517--endometriosislike-syndrome-from-the-concept-of-painful-uterine-syndrome-to-the-use-of-myometrialbotulinum-toxin-injections-under-hysteroscopy

\section{Hosted file}

Figure 1.docx available at https://authorea.com/users/326707/articles/454517--endometriosislike-syndrome-from-the-concept-of-painful-uterine-syndrome-to-the-use-of-myometrialbotulinum-toxin-injections-under-hysteroscopy 